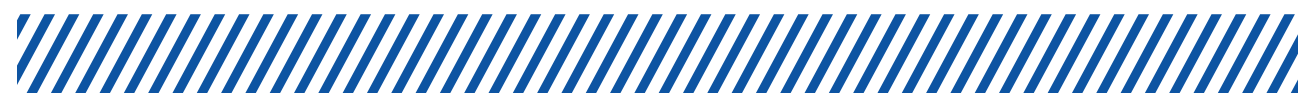

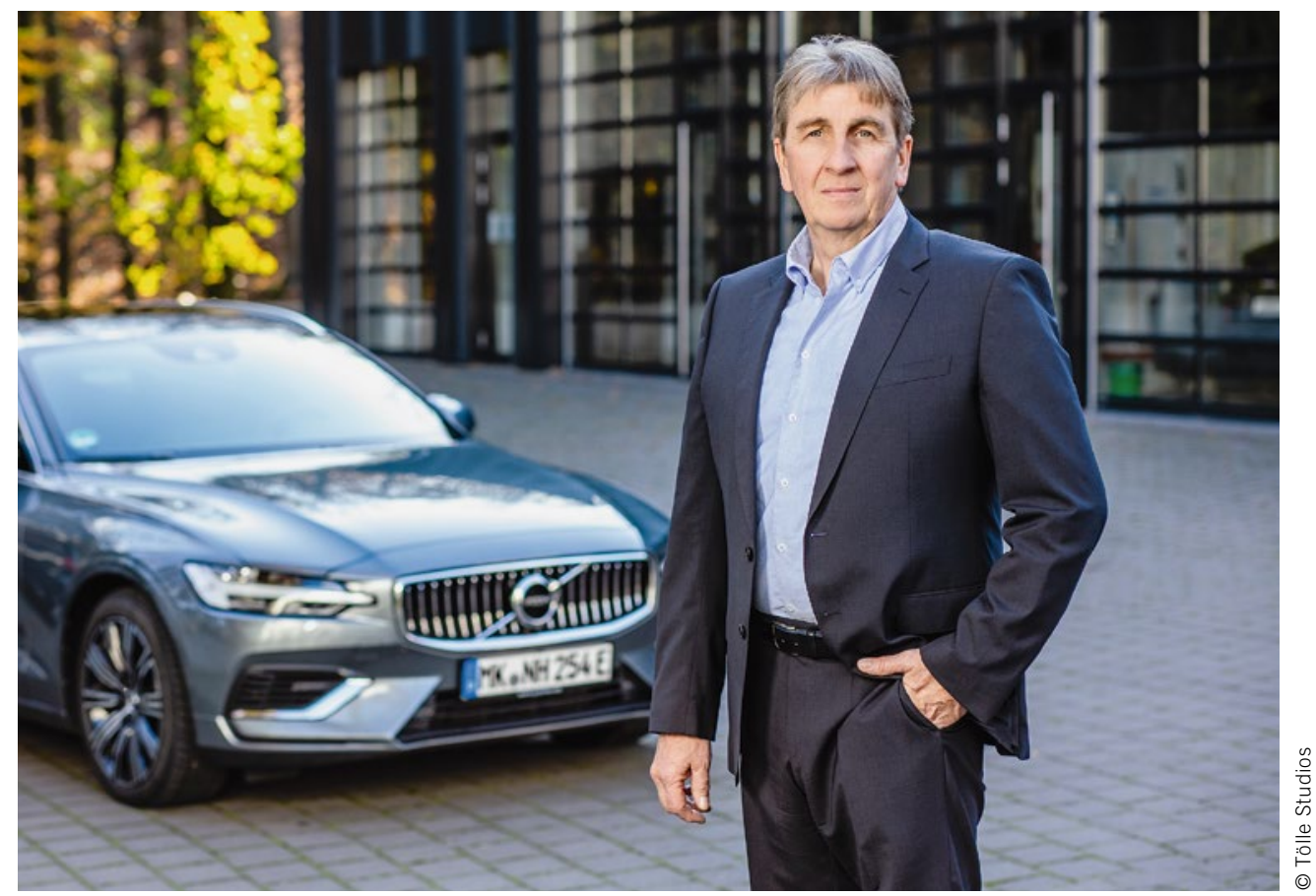

Prof. Dr.-Ing. Wilhelm Hannibal Spokesman for the Automotive Engineering course at the University of Applied Sciences Südwestfalen

\title{
Plug-in Hybrids Will Become Mainstream in Passenger Car Drives
}

At the MTZ symposium “Expert-Forum-Powertrain 2020” held in Hanau in October, there was widespread agreement among experts that hybrid drives are the medium and long-term powertrain solution for mass production. Now it is more a question of the degree of electrification, how battery capacity, and which vehicle classes will be hybridized. Determining the $\mathrm{CO}_{2}$ footprint over the entire life cycle of the vehicle would have to lead us to a factual cradle-to-cradle approach, for which the standards for balancing will need to be defined.

The discussion about medium and long-term passenger car drive systems must be approached with an open mind, depending on the application for the markets in question. The plug-in parallel hybrid will gain further ground in the less cost-sensitive premium segment, where the customer appreciates the displayable performance of a vehicle. This is also underlined by the current range of products on the market. Future political and social developments will have a strong influence on this segment, so that control mechanisms are likely to control purely electric driving.

In the small and compact car segment, the average annual mileage in Germany is around 13,500 km. Cost-effective plug-in hybrid systems will be used here, for example in serial hybrids. The combustion engine will then be less complex for cost rea- sons, and since the customer can drive up to $80 \mathrm{~km}$ purely electrically with a $15 \mathrm{kWh}$ battery for an average distance of less than $60 \mathrm{~km}$ per day, the combustion engine efficiency will play a subordinate role. With regenerative fuels available in the long term, this concept will be superior to a BEV: be emission-free in the city centers and cross the Brenner Pass to Lake Garda $\mathrm{CO}_{2}$-neutral on vacation.

Certainly, the variety of parallel existing drive concepts in passenger cars is increasing, but the plug-in hybrid will become mainstream in mass production in the medium term. Asia has already reconsidered its strategy. Now that subsidies for BEVs in China have been discontinued, the combustion engine with all its application scenarios is once again moving into the focus of further development, especially in the volume segment. Korea and China in particular are making enormous efforts in this cost-sensitive market. A patent and market analysis shows that serial and power-split hybrid drives are favored.

The Covid-19 situation has taught us that we have to be highly flexible to meet the challenges. Applied to vehicle drive technology, this means that communication between politics, business and science must be intensified in order to remain competitive on the world market, independent of Asia. 